\title{
Diversity of quorum sensing autoinducer synthases in the Global Ocean Sampling metagenomic database
}

\author{
Margot Doberva ${ }^{1}$, Sophie Sanchez-Ferandin ${ }^{2}$, Eve Toulza ${ }^{3,4}{ }^{\text {, Philippe Lebaron }}{ }^{1}$, \\ Raphaël Lami ${ }^{1, *}$
}

${ }^{1}$ Sorbonne Universités, UPMC Univ Paris 06, CNRS, Laboratoire de Biodiversité et Biotechnologie Marines (LBBM), Observatoire Océanologique, 66650 Banyuls/Mer, France

${ }^{2}$ Sorbonne Universités, UPMC Univ Paris 06, CNRS, Biologie Intégrative des Organismes Marins (BIOM), Observatoire Océanologique, 66650, Banyuls/Mer, France

${ }^{3}$ UPVD Université de Perpignan, UMR 5244, IHPE, 66860 Perpignan cedex 9, France

${ }^{4}$ CNRS, UMR 5244, 2EI, 66860 Perpignan cedex 9, France

\begin{abstract}
Quorum sensing (QS) is a cell-to-cell signalling pathway that allows bacteria to synchronize their genetic expression. It is mediated by autoinducers (AI), including (1) acyl-homoserine lactones (AHLs or AI-1), produced by Proteobacteria using AinS, LuxI and HdtS synthase families and (2) furanosyl-diester-borate (FDB or AI-2), produced by a large range of phylogenetically diverse bacteria and synthetized by the LuxS family. Few data have been collected about the presence and importance of QS in marine waters using culture independent methods. In this study, we examined the presence and the diversity of AI-1 and AI-2 synthases in the Global Ocean Sampling (GOS), a large metagenomic database, covering 68 stations across 3 oceans. We built 4 reference protein databases with maximal phylogenetic coverage containing all known AI synthase sequences to retrieve AI synthases sequences from the GOS metagenomes. We retrieved 29 environmental sequences affiliated to LuxI (synthesizing AI-1), 653 related to HdtS (AI-1), 31 related to LuxS (AI-2) and only one for AinS (AI-1). AI synthases sequences were found in the 3 oceans covered by the GOS cruise and spanned a large phylogenetic diversity. These data revealed a large number of new marine AI sequences, suggesting that QS based on AI-1 diffusion is a widespread mechanism in the marine environment.
\end{abstract}

KEY WORDS: Quorum sensing · Acyl-homoserine lactone synthases · Global Ocean Sampling · Environmental metagenomes

\section{INTRODUCTION}

Quorum sensing (QS) is a cell-to-cell bacterial communication mechanism, allowing bacteria to sense their population density (Nealson 1977) and coordinate their gene expression levels (Bassler 1999, Fuqua \& Greenberg 2002) and physiological activities (Miller \& Bassler 2001). To perform QSbased communication, bacteria produce, secrete and accumulate small hormone-like molecules called autoinducers (AI) in the nearby environment (Fuqua et al. 1994). As cell density increases, the concentration of these AI increases. When a given threshold concentration is reached, targeted bacterial partners can respond to these compounds and modify their gene expression, and thus their behaviour and phenotype (Withers et al. 2001, Bassler 2002). It has been shown that QS systems regulate and synchronize activities like biofilm production (Parsek \& Greenberg 2005, Dickschat 2010), nodulation (Cha et al. 1998, Loh et al. 2002), bioluminescence (Waters \& Bassler 2005), virulence factor production (Smith \& Iglewski 2003) and many others (Diggle et al. 2007). The coordination of bacterial community activities conveys an ecological advantage to the population (Case et al. 2008). 
QS mechanisms are well described in medical or agronomic model bacterial strains (Cha et al. 1998, Loh et al. 2002) known for their pathogenicity (de Kievit \& Iglewski 2000) or bioengineering potential (March \& Bentley 2004, Brenner et al. 2008). By contrast, little attention has been paid to QS in the functioning of natural microbial communities, including in marine waters (Decho et al. 2010). This is because bacterial densities in seawater $\left(10^{5}\right.$ to $\left.10^{6} \mathrm{cells} \mathrm{m}^{-1}\right)$ are typically below the known thresholds that enable QS (Mohamed et al. 2008). However, at a micrometer scale many ecological niches (such as organic matter particles or microalgal blooms) harbour bacterial concentrations compatible with QS (Gram et al. 2002, Mohamed et al. 2008). In support of this hypothesis, the potential for QS in planktonic marine bacteria has been reported in many cultivated strains. These results were acquired either by directly detecting communication compounds or inferred by sequencing genes involved in QS in Proteobacteria (Gram et al. 2002, Wagner-Döbler et al. 2005).

The AI synthases are the key enzymes involved in AI production. It is now well established that the acylhomoserine lactone synthases (AHL or AI-1 synthases) are encoded by 3 groups of genes: luxI-like (Engebrecht \& Silverman 1984), ainS-like (Gilson et al. 1995) and hdtS-like (Laue et al. 2000, Burton et al. 2005, Rivas et al. 2007). By contrast, luxS-like genes encode the $(2 S, 4 S)$-2-methyl-2,3,3,4-tetrahydroxytetrahydrofuran-borate synthase or $S$-THMF-borate synthase or furanosyl diester borate synthase or $(2 R, 4 S)$-2-methyl-2, 3, 3, 4-tetrahydroxytetrahydrofuran synthase or $R$-THMF synthase, also known as the AI-2 synthases (Xavier \& Bassler 2003, Miller et al. 2004). The AI-1 synthases have been identified in cultivated marine strains from the groups Roseobacter and Vibrio (Gram et al. 2002, Schaefer et al. 2002, Wagner-Döbler et al. 2005) and the AI-2 (Chen et al. 2002) have been observed in the Vibrio genus (Bassler et al. 1997, Bassler 1999). By contrast, very little attention has been paid to the diversity of AI synthases in whole marine microbial communities. As only $1 \%$ of marine bacterial communities in the water column are readily cultivable by standard methods (Amann et al. 1995), an overview of potential QS mechanisms in the marine environment remains largely incomplete.

The Global Ocean Sampling (GOS) database is a large collection of marine metagenomes from 68 sampling sites. A total of 6.3 billion bp were Sanger sequenced from microplankton samples (Rusch et al. 2007) from which the metaproteome (all coding sequences) was also predicted (Yooseph et al. 2007).
The GOS datasets mainly comprise sequences from the free-living fraction (FL) of bacterioplankton $(0.1$ to $0.8 \mu \mathrm{m}$ pore size filter). Additionally, for 8 stations the particle-attached fraction (PA) $(0.8$ to $3 \mu \mathrm{m})$ has been sequenced (GS000, GS001, GS048, GS108, GS110, GS112, GS117 and GS122). The GOS offers a large snapshot of the diversity and functional potential of marine microbial communities and has been successfully explored, for example, for the potential to utilize selenium (Zhang \& Gladyshev 2008) or to metabolize various chemical forms of iron (Toulza et al. 2012).

In this study, we investigated the diversity patterns of QS AI synthases in the bacterial communities represented in the GOS metagenomes. We report the presence, the large diversity of both AI-1 and AI-2 synthase sequences and their distribution throughout the 3 oceans covered by the GOS expedition, and we highlight new sequences of AI synthases.

\section{MATERIALS AND METHODS}

\section{Construction of reference databases for AI synthase proteins}

We built a total of 4 databases, one for each family of AI synthases: AinS-like, LuxI-like, HdtS-like and LuxS-like. Following the approach described in Toulza et al. (2012), we first selected protein sequences that have been functionally characterized in previous publications. To improve taxonomic coverage, we added to our reference databases additional sequences annotated as AI synthase from the GenBank non-redundant (NR) database (www.ncbi. nlm.nih.gov/). Each database was aligned using Clustal W (Larkin et al. 2007) in Jalview (Waterhouse et al. 2009) to discard redundant and partial sequences. Our final AI databases comprised 569 LuxI, 30 AinS, 214 HdtS and 297 LuxS annotated protein sequences.

\section{Screening for AI synthases in the GOS metagenome}

The GOS expedition and its subsequent metagenome analysis have been described previously (Rusch et al. 2007, Yooseph et al. 2007). Briefly, 2001 of seawater was collected at 68 different sampling stations and filtered onto 4 different successive filters with a porosity of $20,3,0.8$ and $0.1 \mu \mathrm{m}$. In this work, we discarded 10 stations where no AI sequences 
were found (GS006, GS038-040, GS042-046, GS050). In our study, we also focused on both FL (0.1 to $0.8 \mu \mathrm{m})$ and PA $(0.8$ to $3 \mu \mathrm{m})$ fractions, which constitute the most complete sequence datasets in terms of sequencing effort. The GOS database contains sequences collected from various habitats within the Atlantic, Pacific and Indian Oceans. A total of 11 different habitat types were sampled, including open ocean (23 stations), coastal (22 stations), coral reefs (4 stations), estuary (2 stations) and 7 others (Rusch et al. 2007). Environmental variables were also retrieved from Venter et al. (2004) and Rusch et al. (2007).

The detection of AI-1 and AI-2 synthases from the GOS database (proteins predicted from reads) was conducted by sequence homology searches using the BLAST (Basic Local Alignment Search Tool) algorithm (Altschul et al. 1990) and our AI synthase databases as the query sequences. All our AI protein BLAST searches were performed using the CAMERA portal (http://camera.crbs.ucsd.edu/projects/ details.php?id=CAM_PROJ_GOS) (Sun et al. 2011) using blastall default parameters (Seshadri et al. 2007) (including FL and PA fractions). A Reciprocal Best BLAST Hit (RBH) analysis (Moreno-Hagelsieb \& Latimer 2008) was then performed on the sequences retrieved from the GOS dataset. Briefly, putative environmental AI synthases were searched against NR database and those GOS sequences with a RBH to the same annotated AI synthases were assigned to the gene and designated as most related to that taxon.

We also compared the distribution of AI environmental protein sequences in both the FL and PA fractions from the 8 GOS sampling stations for which these data are available. We pooled all the retrieved AI protein sequences from PA and FL fractions from all 8 sites, and clustered the environmental and annotated protein sequences using CD-HIT (Li \& Godzik 2006, Fu et al. 2012). The clustering parameters were $40 \%$ sequence identity and a word size equal to 2 . The differences in the number of environmental sequences affiliated to HdtS between the FL and PA fractions were tested for significance using a Wilcoxon test with R (www.r-project.org).

\section{Normalization of AI counts in GOS metagenomes to single copy genes}

The number of sequences matching to AI synthases in the GOS was normalized to the number of RpoB protein sequences present in the sample. That gene is present in only a single copy in bacterial genomes and is not prone to horizontal transfer and thus is also a good taxonomic marker (Case et al. 2007). Following the same procedure as described for the AI sequences, we built an RpoB protein reference databases with a large taxonomic coverage containing 1000 sequences. We finally reported the number of each family of AI environmental sequences related to our reference sequences relative to the number of RpoB protein sequences in the GOS dataset. This allowed normalization to the number of bacterial genomes in the dataset.

\section{Phylogenetic analysis}

Phylogenetic analyses were conducted for each targeted family of AI synthase. All environmental and annotated sequences were aligned using Clustal $\mathrm{W}$ (Larkin et al. 2007) in MEGA v.5.1 software (Tamura et al. 2011), and alignments adjusted manually. Phylogenetic trees were constructed using both Neighbour-Joining (NJ) and Maximum Likelihood (ML) methods for each kind of AI synthase protein sequence alignments. NJ analyses were conducted with gamma correction and 1000 bootstrap replicates. Protein model selection was performed from each protein dataset, and phylogenetic analyses were then conducted with the selected models (WAG model for LuxI, HdtS and LuxS) using gamma correction and 100 bootstrap replicates. As we obtained similar topologies from the 2 methods, only those obtained by NJ are presented here, but when useful, percentage of bootstrap supports obtained by ML searches are also mentioned.

\section{RESULTS}

\section{Features of AI synthase protein reference databases}

All our reference databases were built to have as broad a taxonomic coverage as possible, consisting of protein sequences assignable to AI synthases from the NR database at the time this study was conducted. The LuxI, LuxS and HdtS-like protein family databases had a large taxonomic coverage, which included the alpha-, beta-, gamma- and deltalineages of Proteobacteria. The LuxS protein reference database also contained annotated sequences from Spirochaetes, Bacteroidales and the Gram-positive Firmicutes and Actinobacteria. The AinS family, 
Table 1. Protein families and features of environmental sequences

\begin{tabular}{|c|c|c|c|c|c|}
\hline \multirow[b]{2}{*}{ Features } & \multicolumn{5}{|c|}{ Protein families } \\
\hline & AinS & LuxI & HdtS & LuxS & RpoB \\
\hline Number of annotated sequences & 30 & 569 & 214 & 297 & 1000 \\
\hline Conservation (\%) & 34 & 27 & 27 & 78 & 62 \\
\hline Average number of amino acids & 380 & 200 & 250 & 170 & 1200 \\
\hline Taxonomic affiliation & $\begin{array}{l}\text { Vibrio- } \\
\text { naceae }\end{array}$ & $\begin{array}{l}\text { Proteo- } \\
\text { bacteria }\end{array}$ & $\begin{array}{l}\text { Alpha-, Beta- } \\
\text { and Gamma- } \\
\text { proteobacteria }\end{array}$ & $\begin{array}{c}\text { All bacterial } \\
\text { phyla }\end{array}$ & $\begin{array}{c}\text { All bacterial } \\
\text { phyla }\end{array}$ \\
\hline $\begin{array}{l}\text { Number of Global Ocean Sampling (GOS) } \\
\text { stations where sequences were found }\end{array}$ & $1 / 58$ & $9 / 58$ & $57 / 58$ & $3 / 58$ & $58 / 58$ \\
\hline $\begin{array}{l}\text { Total number of environmental sequences } \\
\text { retrieved in GOS }\end{array}$ & 1 & 29 & 653 & 31 & 4293 \\
\hline $\begin{array}{l}\text { Ratio: number of environmental sequences to } \\
\text { number of RpoB copies (\%) }\end{array}$ & 0.02 & 0.7 & 14.8 & 0.7 & 100 \\
\hline
\end{tabular}

limited to the Vibrionaceae, contained not only AinS but also LuxM and VanM synthases (AinS-like synthases). A total of 634 annotated protein sequences from the National Center for Biotechnology Information (NCBI) were affiliated to LuxI with a mean identity of $27 \%$ (number of conserved amino acids divided by total amino acids), 214 sequences to HdtS with a mean identity of $27 \%, 297$ sequences to LuxS with a mean identity of $78 \%$ and 30 sequences to the AinS with a mean identity of $34 \%$ (Table 1). The mean length of these sequences was about 380 amino acids for AinS, 200 for LuxI, 250 for HdtS and 170 for LuxS.

\section{Abundance and diversity of LuxI synthases in the GOS dataset}

We retrieved 29 sequences related to LuxI AHL synthases in the GOS dataset. Those sequences were distributed over 10 stations of the GOS cruise (Table 1, Fig. 1) and appeared related to a large taxonomic diversity of AI-1 sequences in NCBI. These GOS sequences were aligned with annotated sequences and phylogenetic reconstruction was performed (Fig. 2). All affiliated environmental sequences arose from Alphaproteobacteria (Fig. 2). A total of 19 environmental sequences $(65 \%)$ matched with sequences belonging to Rhodobacterales (Rhodobacteraceae family), 2 (7\%) with sequences belonging to Sphingomonadales (Sphingomonadaceae family) and $3(14 \%)$ with sequences belonging to Rhizobiales (Bradyrhizobiaceae and Beijerinckiaceae families) (Fig. 2). These affiliations were supported by strong
NJ bootstrap percentages (>99 for Rhodobacterales; 100 for Sphingomonadales; 97 and 100 for Rhizobiales; Fig. 2) and also in some cases by strong ML bootstrap percentages. Although well identified as AI-1 synthases by $\mathrm{RBH}$, a large fraction of environmental sequences (14\%) could not be unambiguously related to any annotated sequences.

LuxI sequences arising from PA fractions represented $10 \%$ of total retrieved LuxI sequences in the GOS dataset. These sequences were present at Stns GS048 and GS110. All these sequences appear enclosed in a larger phylogenetic group (defined with bootstraps values of 53/86 for NJ/ML, respectively; Fig. 2) comprising members of the Sphingomonodaceae family.

\section{Abundance and diversity of HdtS synthases in the GOS dataset}

A total of 653 environmental sequences related to HdtS were retrieved from the GOS metagenomes (Table 1). Those sequences were found in all oceans crossed by the GOS expedition and in a large number of stations (57 different stations from a total of 58 sampled by the expedition) (Fig. 1). Collectively, all of these sequences were related to Alpha-, Beta- and Gammaproteobacteria. More precisely, a large fraction (19\% or 127 sequences) of these sequences were related to the SAR11 group (Alphaproteobacteria, with bootstrap supports of 100 and 96 for NJ and ML, respectively), including its cultivated representative member Pelagibacter ubique (indicated by 'SAR11' sequences; 


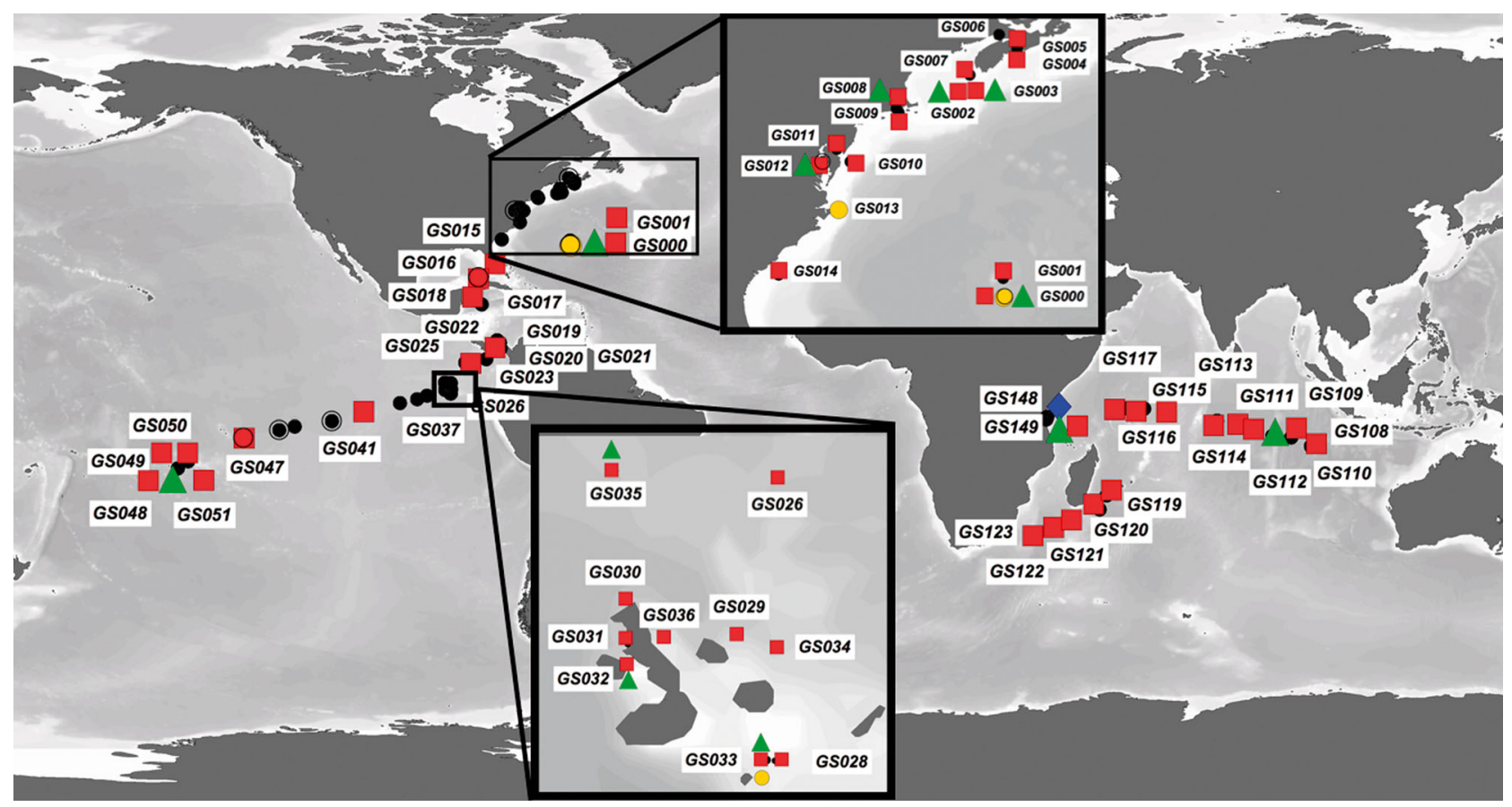

Fig. 1. Geographic distribution of AI-1 (LuxI, HdtS and AinS-like sequences) and AI-2 (LuxS sequences) retrieved from the Global Ocean Sampling (GOS) metagenomes. Black dots: GOS sampling stations where no AI sequences were found; blue diamonds: AinS affiliated sequences; green triangles: LuxI affiliated sequences; red squares: HdtS affiliated sequences; yellow dots: LuxS affiliated environmental sequences. To display the geographic distribution of the environmental sequences related to the 4 different protein families (AinS, LuxI, HdtS and LuxS) a geographic map was built using Ocean Data View software v.4 (ODV Schlitzer, R, Ocean Data View, http://odv.awi.de, 2013)

Fig. 3). A lower fraction (3\% or 21 sequences) of these sequences was related to the SAR116 group (Alphaproteobacteria, with bootstrap supports of 95 and 67 for NJ and ML, respectively; Fig. 3). Interestingly, one sequence was strongly associated to a sequence from Oceanicaulis (Rhodo-bacterales), which belongs to the Hyphomonadaceae family (bootstrap supports of 100 and 83 for NJ and ML, respectively). Among Gammaproteobacteria, a large fraction of sequences were related to the SAR86 group (23\% or 153 sequences) with high bootstrap supports both in NJ and ML methods (100 and 62, respectively; Fig. 3). One sequence was related to Pseudomonadales, and a large fraction ( $21 \%$ or 140 sequences) appeared more closely related to other orders within Gammaproteobacteria (Oceanospirillales, Chromatiales, Acidithiobacillales and Triotrichales). Lastly, a significant number of sequences appeared closely related to the Betaproteobacteria (represented by the Burkholderiales order, $19 \%$ or 126 sequences) and unclassified Gammaproteobacteria $(5 \%$ or 32 sequences) with relatively strong bootstrap supports (63 and 57 for NJ and ML, respectively; Fig. 3).
A few environmental sequences (19\%) were recovered from the 8 sampling stations covering both the FL and PA fractions. Overall, a total of 63 sequences were recovered from the PA fraction. Interestingly, the 2 sequences GS051 FL and GS117 PA were more closely related to (NJ bootstrap support of 98), and found within, the SAR11 Alphaproteobacteria group. Six sequences from the PA fraction of the GS110 sampling site were recovered together and closely related to the Gammaproteobacteria, Francisella tularensis (NJ bootstrap support of 97; Fig. 3). To test whether there was any difference in the taxonomic composition between the FL and the PA fractions, we clustered all HdtS sequences using a threshold of $40 \%$ sequence identity, and found a total of 14 clusters (Table 2). As an example, cluster 6 contains environmental sequences related to SAR86 clade in the 2 fractions of the GOS Stns GS000, GS110 and GS112 (Table 2). In the environmental sequences, of which $11 \%$ were from the FL fraction and $15 \%$ from the PA fraction, we did not find any significant difference between the cluster affiliations of environmental sequences distributed in the 2 metagenome fractions (Wilcoxon test, $\mathrm{p}=0.83$ ). 


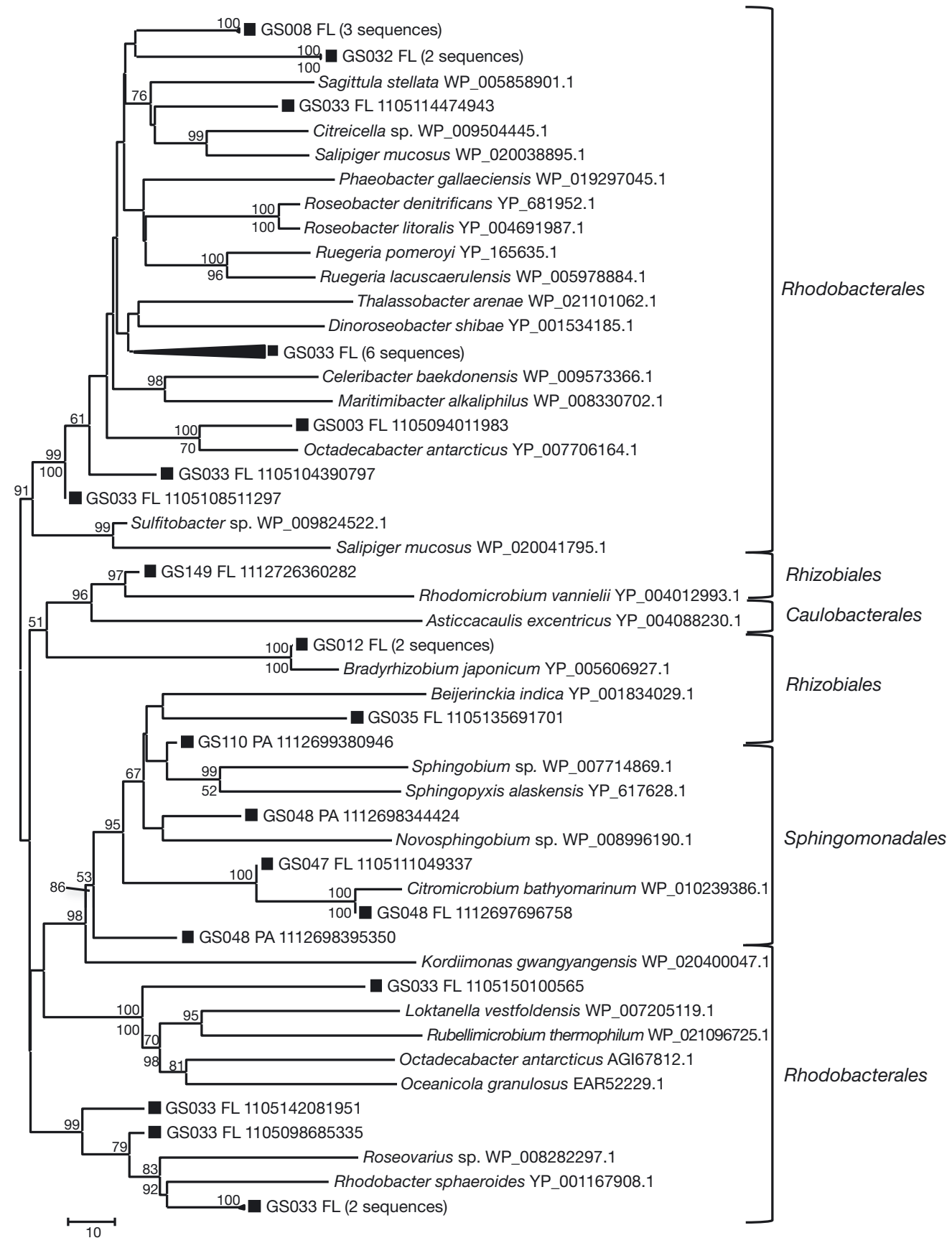

Fig. 2. Phylogenetic tree of annotated and environmental sequences predicted from Global Ocean Sampling (GOS) metaproteome LuxI protein sequences produced using the Neighbour-Joining method with 1000 bootstrap replicates. Only bootstrap support $>50$ are shown. Values above branches are those obtained from NJ method; values under branches are those obtained from ML methods. Black squares: environmental sequences; FL: sequences retrieved from free-living fractions; PA: sequences retrieved from particle-attached fractions

\section{Abundance and diversity of LuxS synthases in the GOS dataset}

A total of 31 environmental sequences related to LuxS were detected in the GOS dataset at 3 sampling sites: GS000, GS013 and GS033 (Table 1, Fig. 1). All environmental sequences affiliated to LuxS were retrieved from the FL fractions. As previously noted, the LuxS family showed the widest taxonomic coverage compared to the other families. The environmental sequences found were related to Gammaproteobacteria $(97 \%$ or 30 sequences, NJ and ML bootstraps support of 100 and $99 \%$, respectively; Fig. 4) and to the Gram-positive bacteria from the 


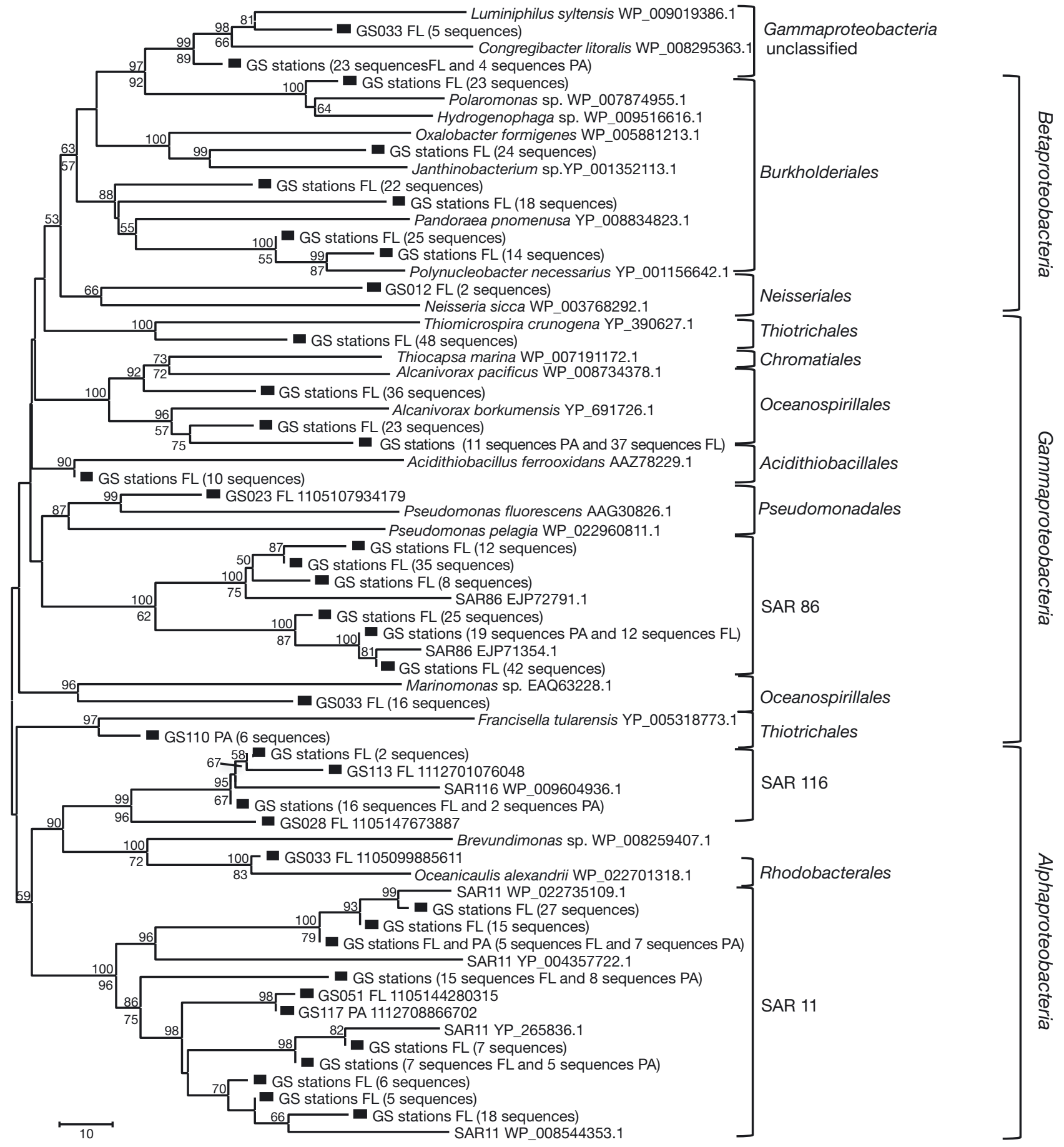

Fig. 3. Phylogenetic tree of annotated and environmental HdtS protein sequences produced using the Neighbour-Joining method with 1000 bootstrap replicates. Only bootstrap support > 50 are shown. Values above branches are those obtained from NJ method; values under branches are those obtained from ML methods. See Fig. 2 for definitions of symbols

Deinococcales (3\% or 1 sequence, NJ and ML bootstraps support of 100 and $90 \%$, respectively; Fig. 4). Among Gammaproteobacteria, 4 sequences (13\%) group inside a clade found in both NJ and ML phylogenies, comprising sequences from Alishewanella and Rheinheimera species (ML bootstrap support of $92 \%$; Fig. 4), but which were more closely related to
Reinheimera (NJ and ML bootstraps support of 93 and $91 \%$, respectively; Fig. 4). A large number of environmental sequences (65\% or 20 sequences) seemed related to Shewanella species, although it was not well supported (except 3 GS000 FL sequences with Shewanella frigidimarina, ML bootstrap support of $60 \%$ ). 
Table 2. Comparison of HdtS phylogenetic cluster abundances (in \% of total sequences) in the free-living (FL) and particle-attached (PA) fractions over 8 Global Ocean Sampling (GOS) stations. HdtS sequences were clustered with CD-HIT software at $40 \%$ identity. Statistical analysis on these data revealed no significant difference of HdtS diversity between the 2 fractions (see 'Materials and methods' and 'Results' for details)

\begin{tabular}{|llrr|}
\hline \multirow{2}{*}{$\begin{array}{l}\text { Cluster } \\
\text { no. }\end{array}$} & Phylogenetic affiliation of & \multicolumn{3}{c|}{ Fraction (\%) } \\
& protein sequence clusters & FL & PA \\
\hline 1 & Luminiphilus/Congregagibacter & 0 & 3.1 \\
2 & Alcalinivorax & 7.1 & 8.7 \\
3 & Oceanocaulis/SAR116 & 3.1 & 1.6 \\
4 & Roseobacter clade & 0.8 & 0 \\
5 & Hahella/Methyloglobulus & 7.1 & 5.5 \\
6 & SAR11 & 8.7 & 9.5 \\
7 & SAR86 & 11.1 & 15 \\
8 & & 3.9 & 2.3 \\
9 & & 3.1 & 0.8 \\
10 & & 3.1 & 0.8 \\
11 & & 0 & 1.6 \\
12 & & 0 & 0.8 \\
14 & Non-affiliable environmental & 0.8 & 0 \\
Total & sequences & 0.8 & 0 \\
& & 50 & 50 \\
\hline
\end{tabular}

\section{Diversity of AinS synthases in the GOS dataset}

We retrieved only 1 sequence of AinS in the GOS dataset, located at Stn 148, in the Indian Ocean close to the Zanzibar coast in a fringing reef. The top high scoring BLAST pair was 'N-(3-hydroxybutanoyl)-L-homoserine lactone synthase luxM' (WP $002541906.1) \quad(70 \%$ sequence coverage and $90 \%$ identity) from Grimontia sp., a Vibrionaceae family member.

\section{Correlation with environmental variables}

For the LuxI group, we found no correlation with the environmental data. For HdtS family there was a positive correlation with metagenome size, and negative correlation between chlorophyll (Spearman $\rho=$ $-0.425, \mathrm{p}=0.002$ ), nitrate (Spearman $\rho=-0.553, \mathrm{p}=$ $5.475 \times 10^{-5}$ ), silica concentration (Spearman $\rho=$ $\left.-0.573801, \mathrm{p}=2.481 \times 10^{-5}\right)$, primary production (Spearman $\rho=-0.374, p=0.01$ ) and the number of environmental sequences related to HdtS. A link with LuxS-related environmental sequences and salinity was also observed (Spearman $\rho=0.341, \mathrm{p}=$ 0.020 ). We did not find any correlation with the other environmental variables (temperature, iron concentration, habitat type) (Table 3).
Table 3. Correlation tests between sequencing effort or environmental variables and AI synthase abundance. For each station, we first checked whether the number of environmental sequences was related to metagenome size with the Spearman coefficient. Then, we verified the existence of a significant relationship between environmental variables and the number of environmental sequences retrieved for each AI family, normalized by the number of potential bacterial genomes at each Global Ocean Sampling (GOS) station (assessed by RpoB, see 'Materials and methods' for details) using a Spearman correlation test (discrete quantitative variables, non-normal). For sequence-habitat correlations, a Kruskal-Wallis test was used (non-parametric, qual itative data against quantitative)

\begin{tabular}{|c|c|c|c|}
\hline Variable & HdtS & LuxI & LuxS \\
\hline \multicolumn{4}{|c|}{ Station metagenome size } \\
\hline Observed stat & 10795.42 & 29835.53 & 21963.30 \\
\hline $\mathrm{p}$-value & $4.425 \times 10^{-8}$ & 0.8070 & 0.0297 \\
\hline$\rho$ & 0.6501 & 0.0331 & 0.2882 \\
\hline \multicolumn{4}{|l|}{ Temperature } \\
\hline Observed stat & 25650.83 & 28923.05 & 29455.69 \\
\hline p-value & 0.5881 & 0.7531 & 0.6497 \\
\hline$\rho$ & 0.0746 & -0.0434 & -0.0626 \\
\hline \multicolumn{4}{|l|}{ Salinity } \\
\hline Observed stat & 17155.63 & 17576.5 & 10683.97 \\
\hline p-value & 0.7018 & 0.579 & 0.0203 \\
\hline$\rho$ & -0.0580 & -0.0840 & 0.3411 \\
\hline \multicolumn{4}{|l|}{ Chlorophyll } \\
\hline Observed stat & 27924.82 & 16952.69 & 17674.31 \\
\hline p-value & 0.0023 & 0.3548 & 0.5018 \\
\hline$\rho$ & -0.4247 & 0.1350 & 0.0967 \\
\hline \multicolumn{4}{|l|}{$\mathrm{Fe}$} \\
\hline Observed stat & 18117.4 & 21233.64 & 15623.73 \\
\hline p-value & 0.7512 & 0.1238 & 0.5179 \\
\hline$\rho$ & -0.0474 & -0.2277 & 0.1239 \\
\hline \multicolumn{4}{|l|}{$\mathrm{NO}_{3}$} \\
\hline Observed stat & 26867.15 & 15388.56 & 14099.07 \\
\hline p-value & $5.475 \times 10^{-5}$ & 0.4605 & 0.34 \\
\hline$\rho$ & -0.5534 & 0.1102 & 0.1848 \\
\hline \multicolumn{4}{|l|}{$\mathrm{Si}$} \\
\hline Observed stat & 27220.46 & 12747.96 & 16131.44 \\
\hline p-value & $2.481 \times 10^{-5}$ & 0.0741 & 0.6529 \\
\hline$\rho$ & -0.5738 & 0.2629 & 0.0673 \\
\hline \multicolumn{4}{|c|}{ Primary production } \\
\hline Observed stat & 23759.31 & 13477.96 & 14815.8 \\
\hline p-value & 0.0097 & 0.1359 & 0.3363 \\
\hline$\rho$ & -0.3737 & 0.2207 & 0.1434 \\
\hline \multicolumn{4}{|l|}{ Habitat type } \\
\hline$\chi^{2}$ & 235.216 & 239.168 & 172.158 \\
\hline p-value & 0.0737 & 0.0665 & 0.3061 \\
\hline $\mathrm{df}$ & 15 & 15 & 15 \\
\hline
\end{tabular}

\section{DISCUSSION}

Our study revealed the presence of AI-1 and 2 synthases, key enzymes in QS pathways, within the GOS predicted metaproteome. To date, QS genes have only been directly found in cultured marine 


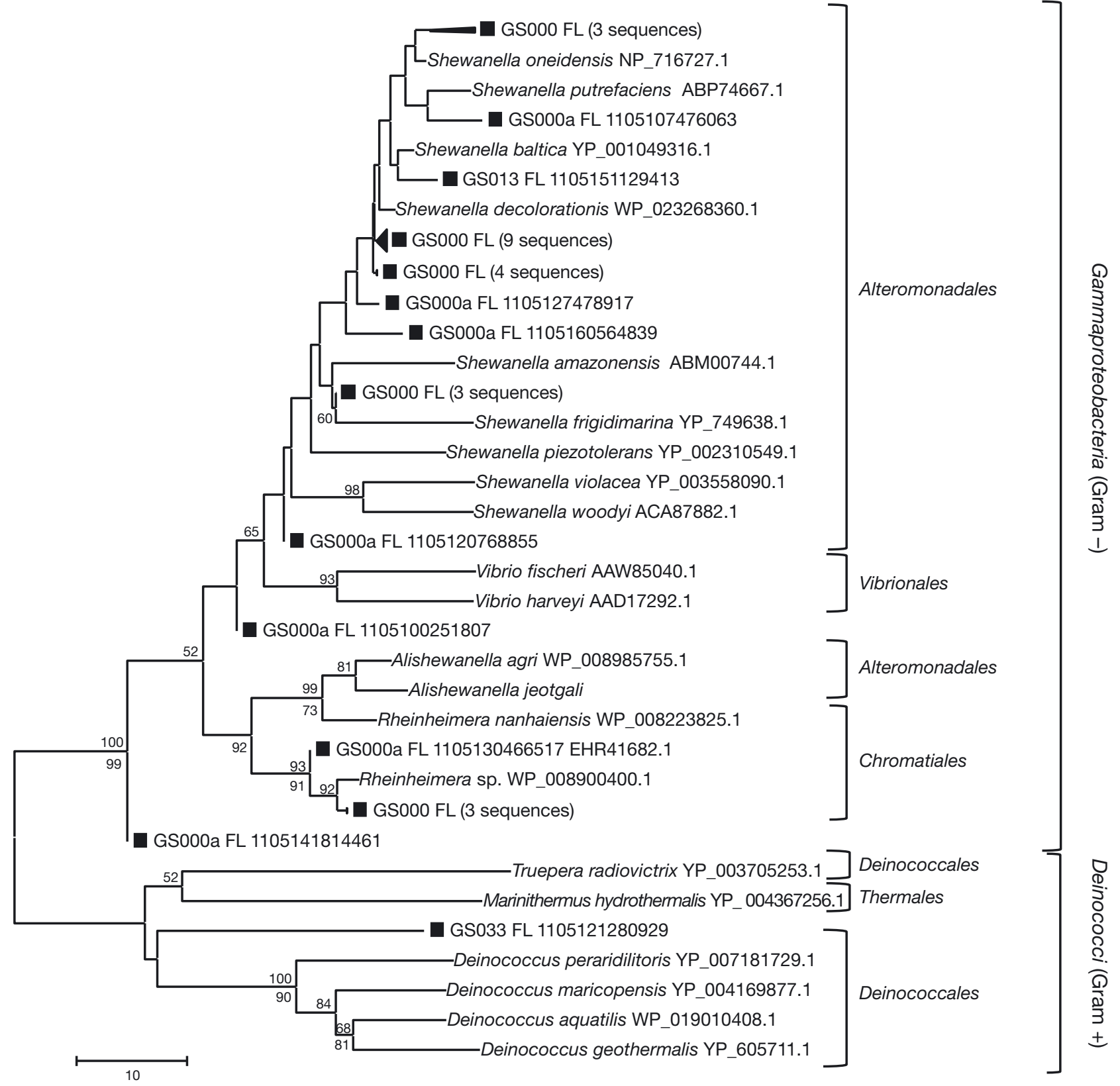

Fig. 4. Phylogenetic tree of annotated and environmental LuxS protein sequences produced using the Neighbour-Joining method with 1000 bootstrap replicates. Only bootstrap support > 50 are shown. Values above branches are those obtained from NJ method; values under branches are those obtained from ML methods. See Fig. 2 for definitions of symbols

bacteria (Engebrecht et al. 1983, Gilson et al. 1995, Gram et al. 2002, Wagner-Döbler et al. 2005) or indirectly inferred from AHL production and genome sequence annotation (Mohamed et al. 2008, Cuadrado-Silva et al. 2013, Doberva et al. 2014a,b). By contrast, no previous studies have examined the presence and diversity of those genes in the marine water column using culture-independent approaches.
We detected a large phylogenetic diversity of AI synthases in the GOS metagenomic libraries. However, it has not been possible to affiliate a large fraction of the retrieved sequences at a fine phylogenetic scale, as only a few marine cultivated strains harbour AI synthase genes that would allow a more accurate taxonomic association. At a higher phylogenetic level, the AI sequences retrieved from the GOS were affiliated to many different groups of bacteria. LuxI 
environmental sequences were mainly related to Rhodobacterales (Cude \& Buchan 2013), Shingomonadales and Rhizobiales, while HdtS sequences were mainly related to SAR11 and Pseudomonadales. These results must be very carefully interpreted, as it has been demonstrated that the phylogenetic signal in AI sequences may be affected by large horizontal transfer between bacterial groups (Gray \& Garey 2001, Lerat \& Moran 2004). However, our data clearly increase the currently known diversity of AI sequences. This high percentage of AI sequences without close relatives from known species in the GOS datasets clearly demonstrates that QS potential is present in a much wider diversity of marine bacteria than previously suspected from cultivable marine bacteria.

We may have underestimated the extent of AI diversity present in the GOS databases. Since our approach was based on sequence homology, we retained only the environmental sequences that were close to known and annotated sequences in databases to avoid false positives. Because of the high stringency of the search criteria used in this study (evalue $10^{-50}$ for first BLAST and $10^{-20}$ for $\mathrm{RBH}$ ), it is possible that divergent $\mathrm{AI}$ environmental sequences were missed. This means that all AI sequences we can detect are dependent on the number and diversity of annotated sequences currently available in the NCBI NR database. The diversity of AI synthases is probably much greater than detectable by similarity searching. Despite this, the number of new AI sequences detected in this study was high, even though total diversity is still probably largely underestimated.

Another recurrent bias to assess when working with metagenomic datasets is the potential functional diversity of annotated proteins (Uchiyama \& Miyazaki 2009, Prakash \& Taylor 2012). Our work indicates that the majority of AI-1 synthases in the GOS dataset are related to the $h d t S$ gene family. HdtS is a member of the lysophosphatidic acid acyltransferase family (Laue et al. 2000) and harbours a dual functionality: acylation of lysophosphatidic acid (Cullinane et al. 2005) and AHL synthesis (Laue et al. 2000, Churchill \& Chen 2011). The production of AHL based on HdtS has been well demonstrated experimentally in Pseudomonas fluorescens (Laue et al. 2000), Acidithiobacillus ferooxidans (Rivas et al. 2007) and Nitrosomonas europea (Burton et al. 2005). When expressed in Escherichia coli, HdtS protein enabled the production of 3-OH-C14:1-AHL, C10AHL and C6-AHL (Laue et al. 2000). One possible mechanism is that HdtS could transfer acyl chains from acyl-ACP or acyl-CoA to S-adenosylmethionine to generate AHLs (Cullinane et al. 2005). Although the experimental evidence accumulated from the strains mentioned above indicates a strong link with AHL production, the large diversity of HdtS found in the GOS metagenomic libraries highlights the need to further confirm a role of diverse HdtS-like proteins in QS.

Our results did not reveal any major difference in AI diversity for HdtS between the FL (0.1-0.8 $\mu \mathrm{m})$ and PA $(0.8-3 \mu \mathrm{m})$ fractions. It is commonly thought that bacteria in sea-water occur in low densities and thus AHLs molecules produced by a cell would quickly be diluted before reaching a receiving cell, hampering QS (Hmelo \& Van Mooy 2009). Thus, a recurrent hypothesis is that QS in marine communities may have more chance to occur in particleattached conditions where bacteria could be concentrated, such as on the surface or inside a particle of sinking organic matter (Mohamed et al. 2008, Hmelo et al. 2011). The absence of a significant difference (Wilcoxon test, $\mathrm{p}=0.83$ ) in HdtS diversity distribution between PA and FL fractions is not surprising as marine bacterial groups can present both FL and PA lifestyles (Ghiglione et al. 2007, Crespo et al. 2013). As the presence of QS genes is similar between PA and FL microenvironments, future studies should focus on detecting differential expression of these genes. Interestingly, recent work conducted on Dinoroseobacter shibae tends to support this hypothesis (Patzelt et al. 2013).

The LuxI and HdtS AI-1 synthase sequences retrieved in this study were detected in metagenomic libraries from the Atlantic, Pacific and Indian Oceans covered by the GOS. This result highlights that AI synthases were present in a wide range of marine environmental conditions, including open and coastal oceans, coral reefs and salt marshes. This observation supports the hypothesis that QS mechanisms may be widespread in many marine environments, and opens the wider question of the potential role of QS in marine prokaryotic species. Furthermore, the negative correlation between environmental sequences related to HdtS and environmental variables linked to coastal marine habitats suggests that HdtS was more prevalent in bacteria living in the open ocean.

Previous studies have revealed a wide phylogenetic distribution of the LuxS group among Proteobacteria, including the marine bacteria genera Vibrio (Bassler 1999) and Shewanella (Bodor et al. 2008), as well as within the Firmicutes, Actinobacteria, Bacteroidales, Deinococcales (Deinococcus) 
and Spirochaetes (Schauder et al. 2001, Lerat \& Moran 2004). The involvement of LuxS in QS remains a matter of debate (see Rezzonico \& Duffy 2008 and Platt \& Fuqua 2010 and references therein). However, many studies also report LuxSbased synthesized molecules as the initiators of a bacterial Esperanto (Winans 2002), i.e. allowing interspecies communication between bacteria. However, our results show a low abundance and a narrow taxonomic coverage of LuxS sequences retrieved from the GOS dataset. This may be due to the lifestyle of bacteria harbouring the AI-2 synthases that are frequently found in association with large organisms (Dworkin \& Falkow 2006, Bodor et al. 2008) and thus may be undetectable with the protocols employed by the GOS cruise. Similarly, AinS AI synthases were very poorly represented in the GOS dataset. This result is perhaps not surprising as these AI synthases are known in only a few members of the Vibrionaceae (Milton et al. 2001), and therefore the low detection rate could be due to the small search database size. Interestingly, Rusch et al. (2007) also did not detect any member of the Vibrionaceae family in the GOS dataset based on 16S rRNA and $r p o B$ gene analysis. This makes it more likely that the low number of AinS detected was due to the low sequence coverage of the GOS metagenomes being unable to detect rare members of the community such as Vibrionaceae. Again, these biases probably underestimated the real extent of marine AI diversity, which we have extended with this study.

Overall, our work has revealed a large number of new AI sequences. Our results show that diverse AI synthase genes are present in the marine environment and that many are related to presently uncultivated bacteria. It appears that AI synthases are diverse and present in all oceans crossed by the GOS expedition, supporting the hypothesis that QS cellto-cell bacterial communication systems are widespread in marine waters. Thus, this study opens the door to the wider questions about the conditions under which expression of these genes occurs and the potential role of QS in marine bacteria and ecosystems.

Acknowledgements. We thank Olivier Naret for his help in reference database constitution. We thank Sheree Yau for ther advice on the revised manuscript version. We are grateful to the CNRS-UPMC ABiMS bioinformatics platform (http://abims.sb-roscoff.fr) for providing computational resources and support. This project was supported by an Emergence UPMC grant. The authors declare no conflict of interest.

\section{LITERATURE CITED}

Altschul SF, Gish W, Miller W, Myers EW, Lipman DJ (1990) Basic local alignment search tool. J Mol Biol 215:403-410

> Amann RI, Ludwig W, Schleifer KH (1995) Phylogenetic identification and in situ detection of individual microbial cells without cultivation. Microbiol Rev 59:143-169

Bassler BL (1999) How bacteria talk to each other: regulation of gene expression by quorum sensing. Curr Opin Microbiol 2:582-587

Bassler BL (2002) Small talk: cell-to-cell communication in bacteria. Cell 109:421-424

Bassler BL, Greenberg EP, Stevens AM (1997) Cross-species induction of luminescence in the quorum-sensing bacterium Vibrio harveyi. J Bacteriol 179:4043-4045

Bodor A, Elxnat B, Thiel V, Schulz S, Wagner-Dobler I (2008) Potential for luxS related signalling in marine bacteria and production of autoinducer-2 in the genus Shewanella. BMC Microbiol 8:13

Brenner K, You L, Arnold FH (2008) Engineering microbial consortia: a new frontier in synthetic biology. Trends Biotechnol 26:483-489

Burton EO, Read HW, Pellitteri MC, Hickey WJ (2005) Identification of acyl-homoserine lactone signal molecules produced by Nitrosomonas europaea strain Schmidt. Appl Environ Microbiol 71:4906-4909

Case RJ, Boucher Y, Dahllöf I, Holmström C, Doolittle WF, Kjelleberg S (2007) Use of 16S rRNA and rpoB genes as molecular markers for microbial ecology studies. Appl Environ Microbiol 73:278-288

Case RJ, Labbate M, Kjelleberg S (2008) AHL-driven quorum-sensing circuits: their frequency and function among the Proteobacteria. ISME J 2:345-349

Cha C, Gao P, Chen YC, Shaw PD, Farrand SK (1998) Production of acyl-homoserine lactone quorum-sensing signals by gram-negative plant-associated bacteria. Mol Plant Microbe Interact 11:1119-1129

> Chen X, Schauder S, Potier N, Van Dorsselaer A, Pelczer I, Bassler BL, Hughson FM (2002) Structural identification of a bacterial quorum-sensing signal containing boron. Nature 415:545-549

> Churchill MEA, Chen L (2011) Structural basis of acylhomoserine lactone-dependent signaling. Chem Rev 111:68-85

> Crespo BG, Pommier T, Fernández-Gómez B, Pedrós-Alió C (2013) Taxonomic composition of the particle-attached and free-living bacterial assemblages in the Northwest Mediterranean Sea analyzed by pyrosequencing of the 16S rRNA. Microbiol Open 2:541-552

> Cuadrado-Silva CT, Castellanos L, Arévalo-Ferro C, Osorno OE (2013) Detection of quorum sensing systems of bacteria isolated from fouled marine organisms. Biochem Syst Ecol 46:101-107

Cude WN, Buchan A (2013) Acyl-homoserine lactone-based quorum sensing in the Roseobacter clade: complex cellto-cell communication controls multiple physiologies. Front Microbiol 4:336

> Cullinane M, Baysse C, Morrissey JP, O'Gara F (2005) Identification of two lysophosphatidic acid acyltransferase genes with overlapping function in Pseudomonas fluorescens. Microbiology 151:3071-3080

de Kievit TR, Iglewski BH (2000) Bacterial quorum sensing in pathogenic relationships. Infect Immun 68:4839-4849

> Decho AW, Norman RS, Visscher PT (2010) Quorum sensing in natural environments: emerging views from microbial 
mats. Trends Microbiol 18:73-80

Dickschat JS (2010) Quorum sensing and bacterial biofilms. Nat Prod Rep 27:343-369

Diggle SP, Crusz SA, Cámara M (2007) Quorum sensing. Curr Biol 17:R907-R910

Doberva M, Sanchez-Ferandin S, Ferandin Y, Intertaglia L and others (2014a) Genome sequence of the spongeassociated Ruegeria halocynthiae strain MOLA R1/13b, a marine Roseobacter with two quorum-sensing-based communication systems. Genome Announc 2:e00993-14

Doberva M, Sanchez-Ferandin S, Ferandin Y, Intertaglia L, Joux F, Lebaron P, Lami R (2014b) Genome sequence of Maribius sp. strain MOLA 401, a Marine Roseobacter with a quorum-sensing cell-dependent physiology. Genome Announc 2:e00997-14

Dworkin M, Falkow S, Rosenberg E, Schleifer KH, Stackebrandt E (eds) (2006) The Prokaryotes, 3rd edn, Vol 6: Proteobacteria: gamma subclass. Springer Science \& Business Media, New York, NY

$>$ Engebrecht J, Silverman M (1984) Identification of genes and gene products necessary for bacterial bioluminescence. Proc Natl Acad Sci USA 81:4154-4158

Engebrecht J, Nealson K, Silverman M (1983) Bacterial bioluminescence: isolation and genetic analysis of functions from Vibrio fischeri. Cell 32:773-781

Fu L, Niu B, Zhu Z, Wu S, Li W (2012) CD-HIT: accelerated for clustering the next-generation sequencing data. Bioinformatics 28:3150-3152

Fuqua C, Greenberg EP (2002) Listening in on bacteria: acyl-homoserine lactone signalling. Nat Rev Mol Cell Biol 3:685-695

Fuqua WC, Winans SC, Greenberg EP (1994) Quorum sensing in bacteria: the LuxR-LuxI family of cell densityresponsive transcriptional regulators. J Bacteriol 176: 269-275

- Ghiglione JF, Mevel G, Pujo-Pay M, Mousseau L, Lebaron P, Goutx M (2007) Diel and seasonal variations in abundance, activity, and community structure of particleattached and free-living bacteria in NW Mediterranean Sea. Microb Ecol 54:217-231

- Gilson L, Kuo A, Dunlap PV (1995) AinS and a new family of autoinducer synthesis proteins. J Bacteriol 177: 6946-6951

Gram L, Grossart HP, Schlingloff A, Kiørboe T (2002) Possible quorum sensing in marine snow bacteria: production of acylated homoserine lactones by Roseobacter strains isolated from marine snow. Appl Environ Microbiol 68: 4111-4116

Gray KM, Garey JR (2001) The evolution of bacterial LuxI and LuxR quorum sensing regulators. Microbiology 147: 2379-2387

Hmelo L, Van Mooy BAS (2009) Kinetic constraints on acylated homoserine lactone-based quorum sensing in marine environments. Aquat Microb Ecol 54:127-133

- Hmelo LR, Mincer TJ, Van Mooy BAS (2011) Possible influence of bacterial quorum sensing on the hydrolysis of sinking particulate organic carbon in marine environments. Environ Microbiol Rep 3:682-688

Larkin MA, Blackshields G, Brown NP, Chenna R and others (2007) Clustal W and Clustal X version 2.0. Bioinformatics 23:2947-2948

Laue BE, Jiang Y, Chhabra SR, Jacob S and others (2000) The biocontrol strain Pseudomonas fluorescens F113 produces the Rhizobium small bacteriocin, N-(3hydroxy-7-cis-tetradecenoyl)homoserine lactone, via
HdtS, a putative novel $N$-acylhomoserine lactone synthase. Microbiology 146:2469-2480

Lerat E, Moran NA (2004) The evolutionary history of quorum-sensing systems in bacteria. Mol Biol Evol 21: 903-913

Li W, Godzik A (2006) Cd-hit: a fast program for clustering and comparing large sets of protein or nucleotide sequences. Bioinformatics 22:1658-1659

> Loh J, Pierson EA, Pierson LS III, Stacey G, Chatterjee A (2002) Quorum sensing in plant-associated bacteria. Curr Opin Plant Biol 5:285-290

- March JC, Bentley WE (2004) Quorum sensing and bacterial cross-talk in biotechnology. Curr Opin Biotechnol 15: 495-502

> Miller MB, Bassler BL (2001) Quorum sensing in bacteria. Annu Rev Microbiol 55:165-199

> Miller ST, Xavier KB, Campagna SR, Taga ME, Semmelhack MF, Bassler BL, Hughson FM (2004) Salmonella typhimurium recognizes a chemically distinct form of the bacterial quorum-sensing signal AI-2. Mol Cell 15:677-687

Milton DL, Chalker VJ, Kirke D, Hardman A, Cámara M, Williams P (2001) The LuxM homologue VanM from Vibrio anguillarum directs the synthesis of $N$-(3-hydroxyhexanoyl)homoserine lactone and $N$-hexanoylhomoserine lactone. J Bacteriol 183:3537-3547

Mohamed NM, Cicirelli EM, Kan J, Chen F, Fuqua C, Hill RT (2008) Diversity and quorum-sensing signal production of Proteobacteria associated with marine sponges. Environ Microbiol 10:75-86

Moreno-Hagelsieb G, Latimer K (2008) Choosing BLAST options for better detection of orthologs as reciprocal best hits. Bioinformatics 24:319-324

> Nealson KH (1977) Autoinduction of bacterial luciferase. Arch Microbiol 112:73-79

> Parsek MR, Greenberg EP (2005) Sociomicrobiology: the connections between quorum sensing and biofilms. Trends Microbiol 13:27-33

> Patzelt D, Wang H, Buchholz I, Rohde M and others (2013) You are what you talk: quorum sensing induces individual morphologies and cell division modes in Dinoroseobacter shibae. ISME J 7:2274-2286

Platt TG, Fuqua C (2010) What's in a name? The semantics of quorum sensing. Trends Microbiol 18:383-387

Prakash T, Taylor TD (2012) Functional assignment of metagenomic data: challenges and applications. Brief Bioinform 13:711-727

Rezzonico F, Duffy B (2008) Lack of genomic evidence of AI2 receptors suggests a non-quorum sensing role for luxS in most bacteria. BMC Microbiol 8:154

> Rivas M, Seeger M, Jedlicki E, Holmes DS (2007) Second acyl homoserine lactone production system in the extreme acidophile Acidithiobacillus ferrooxidans. Appl Environ Microbiol 73:3225-3231

Rusch DB, Halpern AL, Sutton G, Heidelberg KB and others (2007) The Sorcerer II Global Ocean Sampling Expedition: Northwest Atlantic through Eastern Tropical Pacific. PLoS Biol 5:e77

> Schaefer AL, Taylor TA, Beatty JT, Greenberg EP (2002) Long-chain acyl-homoserine lactone quorum-sensing regulation of Rhodobacter capsulatus gene transfer agent production. J Bacteriol 184:6515-6521

Schauder S, Shokat K, Surette MG, Bassler BL (2001) The LuxS family of bacterial autoinducers: biosynthesis of a novel quorum-sensing signal molecule. Mol Microbiol 41:463-476 
Seshadri R, Kravitz SA, Smarr L, Gilna P, Frazier M (2007) CAMERA: a community resource for metagenomics. PLoS Biol 5:e75

Smith RS, Iglewski BH (2003) $P$. aeruginosa quorum-sensing systems and virulence. Curr Opin Microbiol 6:56-60

Sun S, Chen J, Li W, Altintas I and others (2011) Community cyberinfrastructure for Advanced Microbial Ecology Research and Analysis: the CAMERA resource. Nucleic Acids Res 39:D546-D551

Tamura K, Peterson D, Peterson N, Stecher G, Nei M, Kumar S (2011) MEGA5: Molecular Evolutionary Genetics Analysis using maximum likelihood, evolutionary distance, and maximum parsimony methods. Mol Biol Evol 28:2731-2739

Toulza E, Tagliabue A, Blain S, Piganeau G (2012) Analysis of the Global Ocean Sampling (GOS) project for trends in iron uptake by surface ocean microbes. PLoS ONE 7: e30931

Uchiyama T, Miyazaki K (2009) Functional metagenomics for enzyme discovery: challenges to efficient screening. Curr Opin Biotechnol 20:616-622

> Venter JC, Remington $\mathrm{K}$, Heidelberg JF, Halpern AL and others (2004) Environmental genome shotgun sequencing of the Sargasso Sea. Science 304:66-74

> Wagner-Döbler I, Thiel V, Eberl L, Allgaier M and others (2005) Discovery of complex mixtures of novel long-

Editorial responsibility: Craig Carlson, Santa Barbara, California, USA chain quorum sensing signals in free-living and hostassociated marine Alphaproteobacteria. ChemBioChem 6:2195-2206

Waterhouse AM, Procter JB, Martin DMA, Clamp M, Barton GJ (2009) Jalview version 2-a multiple sequence alignment editor and analysis workbench. Bioinformatics 25: 1189-1191

> Waters CM, Bassler BL (2005) QUORUM SENSING: cell-tocell communication in bacteria. Annu Rev Cell Dev Biol 21:319-346

Winans SC (2002) Bacterial Esperanto. Nat Struct Biol 9: 83-84

Withers H, Swift S, Williams P (2001) Quorum sensing as an integral component of gene regulatory networks in Gram-negative bacteria. Curr Opin Microbiol 4:186-193

Xavier KB, Bassler BL (2003) LuxS quorum sensing: more than just a numbers game. Curr Opin Microbiol 6: 191-197

Yooseph S, Sutton G, Rusch DB, Halpern AL and others (2007) The Sorcerer II Global Ocean Sampling expedition: expanding the universe of protein families. PLoS Biol 5:e16

> Zhang Y, Gladyshev VN (2008) Trends in selenium utilization in marine microbial world revealed through the analysis of the Global Ocean Sampling (GOS) project. PLoS Genet 4:e1000095

Submitted: August 22, 2014; Accepted: November 22, 2014 Proofs received from author(s): January 30, 2015 